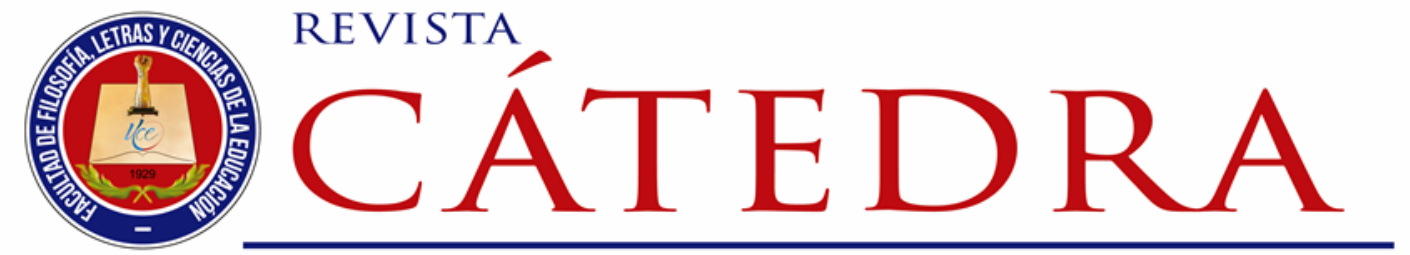

\title{
Investigación y acción participativa: una herramienta metodológica para la comprensión y transformación de la práctica universitaria
}

\section{Participatory research and action: a methodological tool for the understanding and transformation of university practice}

\author{
Jorge Revelo-Rosero \\ Universidad UTE, Quito, Ecuador \\ jorge.revelo@ute.edu.ec \\ https://orcid.org/0000-0002-2756-4856 \\ Sonia Carrillo-Puga \\ Universidad Técnica de Machala, Machala, Ecuador \\ scarrillo@utmachala.edu.ec \\ https://orcid.org/0000-0001-5026-8468 \\ Consuelo Reyes-Cedeño \\ Universidad Técnica de Machala, Machala, Ecuador \\ creyes@utmachala.edu.ec \\ https://orcid.org/0000-0001-7889-5199 \\ Clara Andrade-Erazo \\ Universidad Salamanca, Salamanca, España \\ id00775140@usal.es \\ https://orcid.org/0000-0002-4823-1014
} (Recibido: 18/06/2020; Aceptado: 23/06/2020; Versión final recibida: 15/08/2020)

Cita del artículo: Revelo-Rosero, J., Carrillo-Puga, S., Reyes-Cedeño, C. y Andrade- Erazo, C. (2020). Investigación y acción participativa: una herramienta metodológica para la comprensión y transformación de la práctica universitaria. Revista Cátedra, 3(3), 129-142.

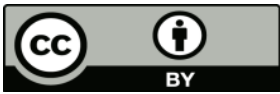




\section{Resumen}

La presente investigación se centra en el estudio de la metodología de Investigación-Acción Participativa (I-AP) como parte de la formación académica de los estudiantes de la Carrera de Educación Inicial. No se evidencian estudios que fundamenten la aplicación de la I-AP a nivel superior, por tanto, es un objetivo de la presente investigación que permita determinar si la I-AP es una metodología que mejora la práctica educativa de los estudiantes de la Carrera de Educación Inicial de la Universidad Técnica de Machala (UTMCH), en vista que, esta metodología hoy en día está dando a la comunidad educativa respuestas que van encaminadas a la solución a los problemas que surgen en la investigación. Dentro de su aplicación se combinan dos procesos importantes en el aula de clase, el de conocer y el de actuar, involucrando en ambos a los estudiantes. Al igual que otros enfoques participativos, ésta proporciona a las comunidades un método para analizar y comprender mejor la realidad de la población, sus problemas, necesidades, capacidades y recursos; $y$, les permite planificar acciones y medidas para transformarla y mejorarla. Se aplicó un diseño de investigación no experimental descriptivo con enfoque cualitativo. Posteriormente, se hizo un análisis descriptivo de la información recogida a través de una entrevista semiestructurada de siete preguntas abiertas. Los resultados obtenidos evidenciaron un mayor interés y expectativa de lo que se les enseñará en la asignatura de Investigación y Acción Participativa: Lesson Study dentro de la Carrera como parte de su formación académica.

\section{Palabras clave}

Investigación educativa, investigación acción participativa, metodología, práctica educativa, sistematización.

\section{Abstract}

This research focuses on the study of the Participatory Action Research (PAR) methodology as part of the academic training of students in the Early Education Career. There is no evidence of studies that support the application of the I-AP at a higher level, therefore, it is an objective of this research to determine whether the I-AP is a methodology that improves the educational practice of students in the Early Childhood Education Career at the Technical University of Machala (UTMCH), given that this methodology today is giving the educational community answers that are aimed at solving the problems that arise in the research. Within its application, two important processes are combined in the classroom, that of knowing and that of acting, involving students in both. Like other participatory approaches, this one provides communities with a method to analyze and better understand the reality of the population, its problems, needs, capacities and resources; and, it allows them to plan actions and measures to transform and improve it. A descriptive, nonexperimental research design with a qualitative approach was applied. Subsequently, a descriptive analysis was made of the information collected through a semi-structured interview of seven open questions. The results obtained showed a greater interest and expectation of what will be taught in the subject of Participatory Research and Action: Lesson Study within the Career as part of their academic training.

\section{Keywords}

Educational research, participatory action research, methodology, educational practice, systematization. 


\section{Introducción}

El presente artículo tiene como objetivo determinar si la metodología de Investigación Acción Participativa incide en la formación académica de los estudiantes de la Carrera de Educación Inicial de la Universidad Técnica de Machala (UTMCH). Teniendo en cuenta que, la I-AP es una metodología activa que permite innovar y mejorar la práctica educativa universitaria, a partir de una relación exitosa de la teoría con la práctica, con el fin de garantizar estándares de calidad que demanda la educación superior para generar conocimiento como una cultura de educación diferente. Al respecto, Revelo-Rosero y Carrillo Puga (2018) consideran que:

toda enseñanza tiene un carácter educativo, ello supone que la educación debe brindar al ser humano no solo condiciones para su formación cognoscitiva, desarrollo de su pensamiento, de sus capacidades y habilidades, sino también para la formación de los distintos aspectos de su personalidad (p. 71).

Por tanto, la educación actual debe dar respuestas a los grandes cambios que ha experimentado la sociedad, debido al alto "impacto de la información tecnológica, la globalización, el crecimiento vertiginoso de las economías generados por la revolución digital" (Revelo-Rosero et al., 2018, p. 199) de las Tecnologías de la Información y la Comunicación (TIC) cada vez más especializadas; hechos que están relacionados con la diversidad de cosmovisiones, identidades, saberes y expresiones a tal punto que ha transformado sustancialmente a lo que se denomina sociedad del conocimiento en un universo complejo y en permanente cambio, es decir, en las formas de enseñar, aprender, comunicar y trabajar.

En las últimas décadas, la investigación en el campo de las ciencias sociales, y de manera especial en las ciencias de la educación, ha producido grades cambios que marcan diferencias significativas en las dimensiones ontológicas, epistemológicas, åéticas y metodológicas, para el acercamiento al objeto de estudio. "Esto implica que estamos ante la presencia de diversos enfoques de investigación que permiten lograr diferentes miradas, ángulos, apreciaciones o valoraciones de una misma situación o tema de estudio" (Colmenares, 2012, p. 102). Por tanto, la investigación ha contribuido de manera sustanciosa a mejorar procesos de innovación que buscan generar cambios que marcan diferencias significativas en el desarrollo de la humanidad, y de modo particular en la docencia universitaria.

En este sentido, en la investigación científica han nacido nuevos enfoques que buscan mayor participación y apropiación del proceso y de los resultados por parte de la comunidad participante, por tanto, en uno de estos nuevos enfoques se ubica la I-AP, lo que, supone un salto cualitativo y cuantitativo que aduce potenciar significativamente los modos de comunicación y generación del conocimiento de los escenarios involucrados. Así, la investigación científica se desarrolla desde la perspectiva de tres enfoques: cuantitativo, cualitativa y mixto, cada uno de ellos involucran diseños de investigación transversales con niveles de estudio de tipo explicativo, descriptivo y correlacional, que contribuyen a la evolución del conocimiento científico desde las aulas universitarias.

El presente trabajo de investigación está estructurado de manera que, en primer lugar, se hace una revisión de la literatura relacionada con conceptos y definiciones relacionados con la I-AP: LS. A continuación, se presenta la metodología utilizada para el diseño de investigación que fue de tipo no experimental descriptivo con un enfoque cualitativo y 
aplicó para la recogida de la información la técnica de la entrevista, seguido de los resultados; y, finalmente se presentan las conclusiones obtenidas. El objetivo de esta investigación es determinar si la Investigación y Acción Participativa: Lesson Study (I-AP: LS) es una metodología que mejora de la práctica educativa de los estudiantes de la Carrera de Educación Inicial.

\section{Revisión de la literatura}

La formación continua del profesorado puede realizarse a través de diferentes modelos que no sólo informan, sino, que además acompañan en los procesos de poner en práctica las innovaciones en el quehacer educativo. En este sentido, la formación del docente, a través de procesos de participación, reflexión y acción, se plantea como fundamental para poder realizar transformaciones innovadoras en el proceso de enseñanza-aprendizaje en las aulas. Del mismo modo, si se hacen de manera colectiva con otros docentes o entre centros educativos, dichas transformaciones no sólo se producirán en el aula, sino que, se darán en todo el contexto educativo.

Para Barba-Martín et al.(2015) una de las formas de poder llevar a cabo una formación continua y colaborativa de los estudiantes, es a través de grupos de Investigación-Acción Participativa, considerándola como un conjunto de prácticas de carácter ético cuyas características permiten que los participantes se formen en función de sus necesidades y, a través del apoyo con otros docentes, con la finalidad de transformar la educación superior en su contexto.

Desde el punto de vista epistemológico, la I-AP plantea primero, que la experiencia les permite a los participantes "aprender a aprender", rompiendo con modelos tradicionales de enseñanza-aprendizaje en los cuales los individuos juegan un papel pasivo, acumulando la información que el profesor les imparte. Segundo, el proceso de investigación les permite a los integrantes de la comunidad aprender cómo conducir la misma y valorar el papel que juega la investigación en su vida. Tercero, los participantes en la I-AP aprenden a entender su papel en el proceso de transformación de su realidad social, como actores centrales en el proceso de cambio. Por último, el promover el desarrollo de conciencia crítica entre los participantes, se convierte en un proceso liberador (Balcázar, 2003).

La I-AP es una metodología de mucha riqueza, ya que, por una parte, permite generar nuevo conocimiento, y por otra, genera respuestas concretas a problemáticas planteadas por los investigadores sobre interrogantes o temáticas de interés que produzcan nuevos conocimientos. Del mismo modo, la I-AP ha sido introducida no solo como una metodología de enfoque cualitativo, sino también de promoción de cambio social, que "busca desarrollar el pensamiento reflexivo, crítico y emancipador para transformar y resolver problemas de una realidad social a través del trabajo constante, colaborativo e interactivo de todos los implicados" (Molina Olavarría, 2015, p. 152). Es decir, que implica una nueva forma de investigar, que conlleva consigo un cambio de actitudes para generar nuevo conocimiento.

Benalcázar y Hernández (2002) consideran a la metodología I-AP como una estrategia para conducir la investigación, ofreciendo pautas que permitan adecuarlas a una situación específica de acuerdo al contexto en el que se implementa la investigación. En este sentido, se han ido implementando modelos de I-AP para que los estudiantes los utilicen como marco de reflexión y acción en la praxis con la finalidad de resolver problemas de la sociedad que se encuentra en constante cambio.

Cáceres, García y Sánchez (2002), Latorre (2003) y Pereyra (2008) entre otros, afirman que la I-AP se caracteriza por ser colaborativa, participativa, democrática, auto evaluativa,

Licencia Creative Commons Atribución 4.0 Internacional (CC BY 4.0)

Revista Cátedra, 3(3), pp. 129-142, septiembre-diciembre 2020. e-ISSN:2631-2875 https://doi.org/10.29166/catedra.v3i3.2187 
interactiva, que permite una retroalimentación continua, facilita una aplicación inmediata, se realiza dentro de un contexto social y contribuye tanto a la ciencia social como al cambio social con una capacidad transformadora tanto del docente como de sus estudiantes. Del mismo modo, para Lewin (1946), Kolb (1984) y, Carr y Kemmis (2003), la metodología IAP es un proceso que está integrado por cuatro fases o momentos interrelacionados entre sí: planificación, acción, observación y reflexión (Ver figura 1).

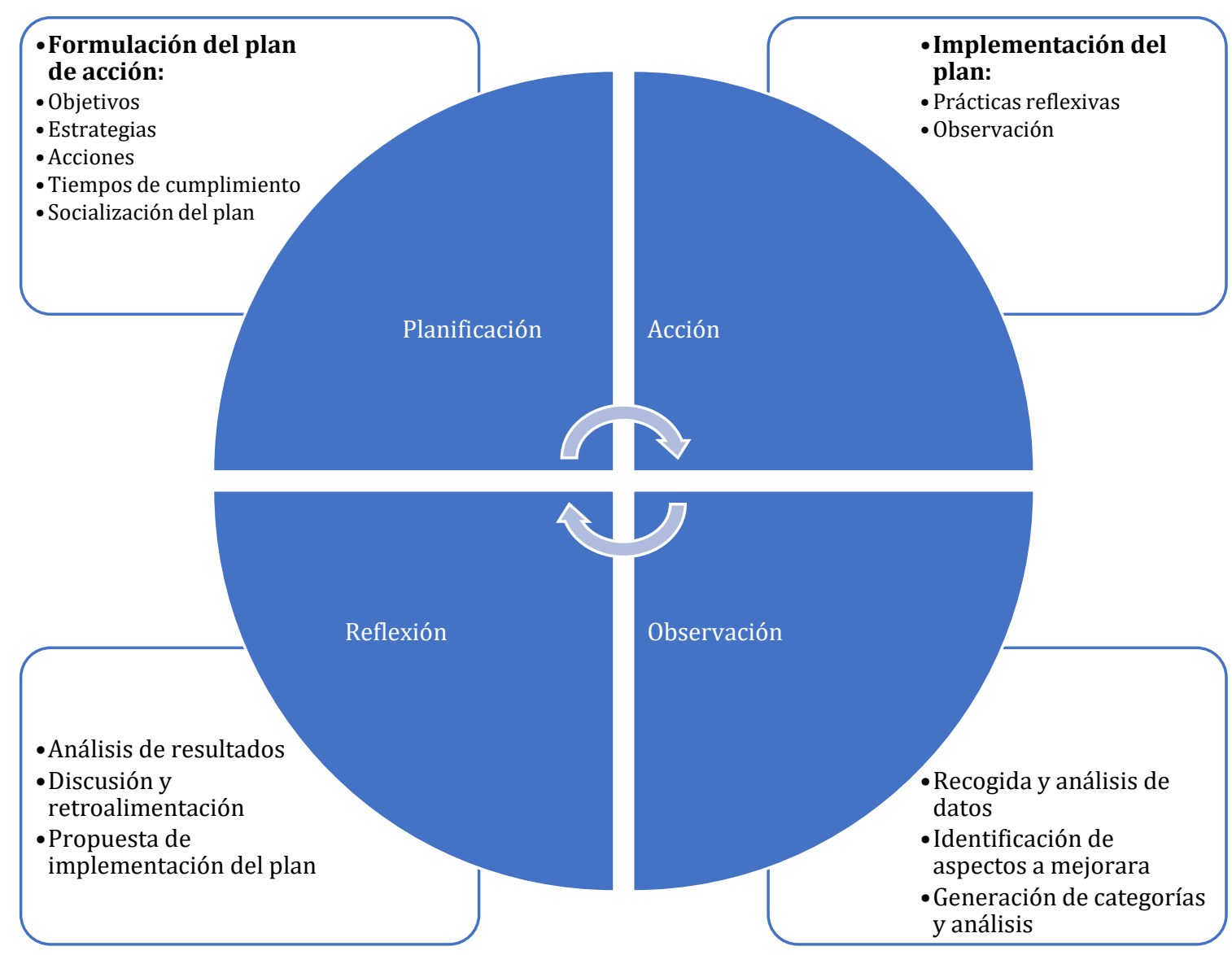

Figura 1. Fases de la metodología I-AP. Adaptado de: (Colmenares, 2012, p. 107)

Soto Gómez y Pérez Gómez (2015) afirman que la Lessons Study (LS) es una metodología de investigación que sustituye a los procesos tradicionales de reflexión y mejora a la práctica educativa, es decir, que aporta a la construcción de saberes y prácticas docentes. La LS tiene su origen y con una amplia difusión en Japón y con gran repercusión en países asiáticos como China, Singapur, Malasia, Indonesia y Tailandia, así como en Norte América, y recientemente en Europa: Suecia y Reino Unido, con pedagogos de reconocida trayectoria internacional, (...). Además, es necesario destacar las aportaciones que realizan estos autores, sobre las características de la LS, que aportan notablemente a mejorar la práctica educativa en países orientales y latinoamericanos, y que actualmente es utilizada en Ecuador en la elaboración de los rediseños de todas las carreras, en las que se destaca la importancia de desarrollar en los estudiantes competencias esenciales para desarrollar procesos de investigación. En otras palabras, la metodología de la LS establece la relación entre un conjunto de prácticas, hábitos, relaciones interpersonales y herramientas que ayudan al docente a trabajar de forma cooperativa en un proceso de acción e investigación centrado en el aprendizaje de los estudiantes que permitan fortalecer el desarrollo de 
competencias profesionales en comunidades de aprendizaje. Al respecto, Greenwood (2000) afirma que "la investigación-acción es una forma de investigación 'co-generativ', en el sentido de que un grupo formado por los dueños legítimos del problema y un investigador profesional se reúnen para codesarrollar sus metas investigadoras y para el cambio social" (p. 33). En el ámbito educativo, es importante el uso forma de investigación, especialmente en situaciones donde se presentan problemas prácticos, incoherencias e inconsistencias entre lo que se persigue y lo que en la realidad se persigue.

La I-AP: LS al ser una herramienta que cuenta con una metodología de investigación que busca relacionar la teoría con la praxis como punto de partida para innovar la educación en todos los niveles, convirtiéndose en el eje de la formación inicial en diferentes espacios del contexto educativo, relacionando de manera cooperativa y colaborativa a docentes y los estudiantes. Por otro lado:

La Investigación - Acción Participativa es una metodología inclusiva que hace participe y protagonista del proceso de enseñanza al alumnado desde el primer día de su formación dando a conocer una estructura que apoya y cohesiona al grupo contribuyendo a la creación de una verdadera comunidad educativa, algo básico en la construcción de la escuela que queremos (Pérez Gómez \& Soto Gómez, 2011, p. 64).

Del mismo modo, Colmenares (2012) afirma que "la I-AP constituye una opción metodológica de mucha riqueza, ya que, por una parte, permite la expansión del conocimiento, y por la otra, genera respuestas concretas a problemáticas que se plantean los investigadores cuando deciden abordar una interrogante" (p. 2). Es importante, destacar que la autora menciona que la Investigación-Acción Participativa es una metodología que hace referencia a procedimientos específicos de un estudio científico diferente a la investigación tradicional, puesto que, es una manera concreta de llevar adelante los pasos de una investigación científica.

En este sentido, la I-AP constituye una alternativa para expandir el conocimiento, apoyada en métodos y técnicas comprobadas para dar claridad a la investigación y precisar sus resultados, así mismo, está generando respuestas fiables a los investigadores acerca de los colectivos investigados, sobre todo, ayuda tomar las acciones necesarias y adecuadas para el cambio y la trasformación de la sociedad.

\subsection{Ventajas e inconvenientes del uso de la metodología}

Investigadores como Díaz Méndez 1992, Pérez 2011, Castañeda 2013, entre otros; establecen algunas ventajas e inconvenientes al usar a la I-AP como enfoque cualitativo, para la recogida y análisis de la información con el fin de dar solución a los problemas planteados dentro de un trabajo de investigación (Ver cuadro 1).

A continuación, se muestra el cuadro 1 que resume algunas ventajas e inconvenientes del uso de la metodología I-AP.

\begin{tabular}{ll}
\hline VENTAJAS & INCONVENIENTES \\
\hline Genera un interesante, amplio y creativo & Toma mucho tiempo de aplicación, y \\
proceso de auto-aprendizaje. & $\begin{array}{l}\text { existen problemas que tienen que ser } \\
\text { solucionados a la brevedad posible. }\end{array}$ \\
$\begin{array}{l}\text { Genera respuestas a los investigadores y al } \\
\text { grupo investigado, para tomar acciones }\end{array}$ & \\
\hline
\end{tabular}

Licencia Creative Commons Atribución 4.0 Internacional (CC BY 4.0)

Revista Cátedra, 3(3), pp. 129-142, septiembre-diciembre 2020. e-ISSN:2631-2875

https://doi.org/10.29166/catedra.v3i3.2187 
adecuadas para el cambio y la En muchos casos puede haber trasformación de la comunidad. manipulación de los resultados debido las

Favorece la recreación de las redes sociales y los lazos de solidaridad.

Desarrolla capacidades participativas y habilidades para el trabajo en equipo y en red, permitiendo aprender, consensuar y negociar.

Permite un alto grado de compromiso y corresponsabilidad por parte de los participantes.

Proporciona una gran aceptabilidad y riqueza propositiva y proyectiva.

Permite describir las relaciones sociales primarias con mayor profundidad y agilidad que otras técnicas.

Permite al investigador no solo conocer una determinada realidad o un problema específico de un grupo sino que desea resolverlo.

Contribuye a la democratización de la acción educativa, social y comunitaria.

Permite la integración masiva de la comunidad.

Mediante la participación de la gente se logra concientizar sobre los problemas existentes dentro de los grupos estudiados, sino, sobre la solución de los mismos.

Permite reflexionar sobre las necesidades reales existentes en la comunidad.

No solo comprende la realidad sino que también, se esfuerza por mejorarla.

Absorbe la mayor cantidad de la información proporcionada por la gente, sus creencias y significaciones.

Es un ciclo de auto-reflexión para los participantes al momento de dar solución a los problemas planteados en la investigación.

Cuadro 1. Ventajas e inconvenientes del uso de la metodología I-AP. Adaptado de: (Pérez, p. 4-5 y Castañeda, 2013, p. 9-10) 
En este contexto, acorde a las necesidades de estudio en la formación de los futuros profesionales de la Carrera de Educación Inicial, se seleccionan actividades, estrategias, técnicas y plantean instrumentos para la recolección de bibliografía necesaria para utilizarla en el momento más apropiado en cada una de las asignaturas que tributan al desarrollo del Proyecto Integrador de Saberes que presentan los estudiantes al finalizar el nivel. Por tanto, se hace imperiosa la necesidad de resaltar que desde la asignatura se favorece el desarrollo de la reflexión, la escritura, redacción, análisis y síntesis en los estudiantes con relación a los procesos que deben cumplir para manejar la investigación científica dentro y fuera del aula; lo que les permitirá desarrollar habilidades y destrezas empezando desde la redacción de informes de investigación, parafrasear, diseñar párrafos descriptivos en temáticas de interés hasta llegar a la escritura académica.

En los procesos de formación integral que se persigue desarrollar en los universitarios, se debe resaltar la Investigación y la Epistemología; la misma que se encuentra enmarcada a lo largo de la Malla Curricular de la Carrera de Educación Inicial de UTMCH en las tres unidades de formación profesional: Unidad Básica, Profesional y de Titulación. En este sentido, la asignatura Investigación y Acción Participativa: Lesson Study (I-AP: LS), se ubicada en la Unidad de Formación Básica, de los estudiantes del Primer Nivel; donde se la presenta con el objetivo de observar los problemas educativos mediante la I-AP: LS, desarrollar la capacidad de prevención de futuras situaciones con el uso del conocimiento. Está organizada en cuatro unidades de aprendizaje, en donde se pone de manifiesto las competencias investigativas del estudiante con el fin de propiciar situaciones de aprendizajes en el desarrollo de habilidades y destrezas, actitudes y valores, en el manejo de la investigación científica.

\section{Materiales y métodos}

El diseño de la investigación es de tipo no experimental descriptivo con un enfoque cualitativo y se enmarcó dentro de la investigación - Acción Participativa que permita relacionar desde perspectiva empírica y conductual, realidad curricular y práctica profesional de los estudiantes de la Carrera de Educación Inicial. Para para la recogida de la información se aplicó la técnica de la entrevista semiestructurada.

La población con la cual se trabajó fueron estudiantes de Primer Nivel, paralelo B la asignatura de Investigación y Acción Participativa: Lesson Study (I-AP: LS) de la Carrera de Educación Inicial del semestre mayo-agosto 2018. Para la selección de la muestra se tomó al total de la población que era de 32 estudiantes, de los cuales $29(90,6 \%)$ eran de género femenino y $3(9,4 \%)$ de género masculino.

A continuación, se muestra el cuadro 2, donde se resume el total de la muestra clasificada por género, frecuencia y porcentaje de participantes masculinos y femeninos.

\begin{tabular}{llllll}
\hline & & Frecuencia & Porcentaje & $\begin{array}{l}\text { Porcentaje } \\
\text { válido }\end{array}$ & $\begin{array}{l}\text { Porcentaje } \\
\text { acumulado }\end{array}$ \\
\hline Válidos & masculino & 3 & 9.4 & 9.4 & 9,4 \\
& Femenino & 29 & 90,6 & 90,6 & 90,6 \\
\hline Total & 32 & 100 & 100 & \\
\hline
\end{tabular}

Cuadro 2. Muestra

Con relación al objetivo propuesto se planteó la siguiente interrogante de investigación: La Investigación y Acción Participativa: Lesson Study (I-AP: LS) es una metodología que mejora 
de la práctica educativa de los estudiantes de la Carrera de Educación Inicial de la Universidad Técnica de Machala.

\subsection{Instrumento para la recolección de información}

Para la recogida de la información se aplicó la técnica de la entrevista, con la aplicación de un cuestionario semiestructurado de siete preguntas abiertas dirigido a los estudiantes el Primer Nivel de la Carrera de Educación Inicial de la Universidad Técnica de Machala en la asignatura de Investigación y Acción Participativa: Lesson Study (I-AP: LS).

En efecto, se proyecta que los estudiantes de la asignatura de I-AP: LS del Primer Nivel de la carrera de Educación Inicial, de la Universidad Técnica de Machala, identifiquen los pasos para la búsqueda de información científica, en la redacción de documentos y textos científicos - académicos desde la práctica y que den respuesta a las necesidades formativas necesarias en su desarrollo profesional.

La metodología diseñada para el efecto, es la de investigación-acción participativa, como una metodología rectora del proceso investigativo. La organización viene dada por unidades previamente organizadas en el nivel, generándose en cada unidad, procesos investigativos. Para lo cual se proyecta que los estudiantes:

- Identifiquen tipos de textos con la lectura: Expositivos, Argumentativos, CientíficosTécnicos y Académicos.

- Aprovechen los espacios de aprendizajes organizados por la docente para mejorar los aprendizajes.

- Jerarquicen las estrategias necesarias para la redacción de ensayos como producto de la investigación.

- Organicen espacios de investigación para la ejecución del Proyecto Integrados de saberes.

- Programen escenarios de escritura académica para su formación profesional.

Para ello, se hace necesario el desarrollo de competencias en los estudiantes de educación superior, la misma va ganando espacios dentro de las aulas universitarias, donde el docente no solo investiga, sino que lo hace junto a sus estudiantes. En este sentido, Rivas (2011, p. 34), menciona que: "vivimos en un mundo feroz, donde ganar competencias se ha vuelto sinónimo de éxito". En este sentido, al impartir la asignatura de Investigación y Acción Participativa: Lesson Study (I-AP: LS) a los estudiantes de Primer Nivel, de la Carrera de Educación Inicial se lograron como parte de su formación académica, los resultados de aprendizaje siguientes:

- Aplica la Historia del Arte en los procesos de investigación.

- Diseña estrategias de lectura comentada, argumentativa, de análisis y síntesis.

- Desarrolla destrezas de escritura y redacción de párrafos.

- Muestra habilidades de análisis, y síntesis al redactar textos científicos.

\section{Resultados}

En base a lo planteado en el apartado anterior, se demuestra que los estudiantes del I Nivel paralelo "B" de la carrera en mención, deciden asumir el compromiso del cumplimiento de cada una de las actividades en los temas y subtemas programados en las cuatro unidades didácticas, lo que les permitirá mejorar los procesos de aprendizaje. De cara a esta problemática, se aplicaron a los estudiantes las siguientes interrogantes:

1. ¿Qué habilidades de búsqueda de literatura científica poseen? 
2. ¿En su formación secundaria, le brindaron la oportunidad de desarrollar habilidades y destrezas investigativas?

3. ¿De 1 a 10, cómo calificaría usted el aprendizaje obtenido en cuanto al desarrollo de la metodología de investigación académica recibida?

4. ¿Sus profesores le facilitaron sitios web donde se podía acceder a información necesaria en su formación?

5. ¿Le enseñaron a redactar, analizar, sintetizar y hacer uso de estos elementos para desarrollar textos de lectura?

6. ¿Le gustaría fortalecer habilidades y destrezas de investigación académica y científica en beneficio de su formación profesional?

7. ¿La asignatura Investigación - ¿Acción Participativa, considera usted que le aportará en su formación profesional?

Resumiendo las interrogantes anteriores se tiene que (Ver cuadro 3):

Cuestionario Análisis de las respuestas de la entrevista

semiestructurado

\begin{tabular}{|c|c|}
\hline Pregunta 1 & $\begin{array}{l}\text { El total de la muestra }(n=32) \text { entrevistada manifestó que en su } \\
\text { formación secundaria no desarrolló habilidades en la búsqueda de } \\
\text { una adecuada literatura científica }(\ldots) \text {. }\end{array}$ \\
\hline Pregunta 2 & $\begin{array}{l}\text { Las dos terceras partes }(\mathrm{n}=21) \text { de los entrevistados afirman que } \\
\text { en el nivel medio, no han desarrollado habilidades y destrezas } \\
\text { investigativas dentro las asignaturas que recibieron }(\ldots) \text {. }\end{array}$ \\
\hline Pregunta 3 & $\begin{array}{l}\text { La mayoría de la muestra }(\mathrm{n}=25) \text { afirma en una escala de } 4 \text {, que el } \\
\text { aprendizaje adquirido en cada una de las asignaturas se debe al } \\
\text { desarrollo de una metodología de investigación científica }(\ldots) \text {. }\end{array}$ \\
\hline Pregunta 4 & $\begin{array}{l}\text { Casi el total }(\mathrm{n}=31) \text { de los entrevistados indicaron que no les } \\
\text { habían facilitado sitios en la web para poder acceder a información } \\
\text { adecuada para su lectura e investigación; frente a un reducido } \\
\text { grupo que indicaron que por su propia cuenta investigaban y } \\
\text { encontraban sitios donde encontraban valiosa información que les } \\
\text { servía de apoyo a las asignaturas que recibían (...). }\end{array}$ \\
\hline Pregunta 5 & $\begin{array}{l}\text { Las dos terceras partes }(\mathrm{n}=21) \text { de los estudiantes entrevistados } \\
\text { indicaron que la institución no les han enseñado frente a un tercio } \\
(\mathrm{n}=11) \text { que manifestaron que si les habían enseñado a redactar, } \\
\text { analizar, sintetizar y hacer uso de estos elementos para desarrollar } \\
\text { textos de lectura (...). }\end{array}$ \\
\hline Pregunta 6 & $\begin{array}{l}\text { El total de la muestra }(\mathrm{n}=32) \text { de los estudiantes señalan que les } \\
\text { gustaría fortalecer sus habilidades y destrezas en la investigación } \\
\text { académica y científica que vayan en formación profesional (...). }\end{array}$ \\
\hline Pregunta 7 & $\begin{array}{l}\text { Del mismo modo, el total de la muestra }(\mathrm{n}=32) \text { señala que se } \\
\text { encuentra muy entusiasmada y manifiestan gran interés y } \\
\text { expectativa de lo que se les enseñará en la asignatura de } \\
\text { Investigación y Acción Participativa: Lesson Study (...). }\end{array}$ \\
\hline
\end{tabular}

Cuadro 3. Resultado de la entrevista a estudiantes de Primer Nivel de educación Inicial

La I-AP, está concebida como un aprender - haciendo, proceso en el cual el investigador debe actuar como facilitador, orientador y como persona técnica a quien se le consulta. Para realizar un proyecto basado en la "Lesson Study", se plantea desde dos perspectivas básicas las cuales son:

Licencia Creative Commons Atribución 4.0 Internacional (CC BY 4.0)

Revista Cátedra, 3(3), pp. 129-142, septiembre-diciembre 2020. e-ISSN:2631-2875

https://doi.org/10.29166/catedra.v3i3.2187 
- Diseño bibliográfico o investigación temática: Este diseño utiliza datos secundarios, este tipo de investigación es teórica por lo que necesitamos las pruebas suficientes para sustentar nuestro proyecto.

- Diseño de campo o investigación empírica: Este diseño utiliza datos primarios que se los obtiene principalmente de la realidad, para la cual se utilizan el Diseño experimental y de Encuestas, como se lo hizo en este ciclo para obtener una información real de la problemática del proyecto que se vaya a realizar.

Se pretende brindar a los estudiantes herramientas adecuadas para mejorar sus aprendizajes en la búsqueda de nuevos conocimientos. Hay que mencionar, que a los estudiantes se les indica que citen los autores y los artículos de los cuales obtuvieron la información, y, por otro lado se necesita que el proyecto sea verificado utilizando métodos como la recolección de datos, una entrevista o una encuesta. "En todos los niveles de educación se requiere que los docentes y estudiantes aprendamos a investigar sobre nuestros propios procesos de enseñanza en el marco de modelos de investigación- acción" (Figueroa Sandoval et al., 2012).

Los estudiantes manifestaron que los conocimientos recibidos y temas planteados, les ayudaron a la realización del proyecto. Es decir, fueron productivos ya que todos investigaron, leyeron, analizaron, razonaron y sintetizaron cada una de las lecturas científicas (artículos), en las cuales se apoyaron para desarrollar temas del proyecto. Situación que les permitió apropiarse de los conocimientos con temas de interés adecuados de su formación académica, y necesaria para mejorar sus procesos cognitivos y poder decodificar, entender e interpretar un texto; convirtiéndose en asiduos lectores que construirán propios aprendizajes significativos con los conocimientos previos utilizando las claves lingüísticas y textuales procedentes del texto.

\section{Discusión y conclusiones}

La I-AP es una metodología que coadyuva al desarrollo de experiencias formativas en los estudiantes de Educación Inicial, bajo el enfoque cualitativo, crítico, reflexivo y transformador, donde cada uno de los estudiantes pudo abordar su participación como resultado de la comprensión de lo que docente impartía en el aula. Situación que les permitió plantear puntos de vista, análisis de sucesos, propuestas de actividades y estrategias que iban directamente ligadas con las temáticas de estudio. Además, Se pudo evidenciar como los estudiantes desarrollaron destrezas para buscar información que le permita desarrollar su proyecto integrador de saberes (PIS), relacionando la experiencia de sus prácticas pre profesionales con la ejecución del mismo, y lo más enriquecedor fue el compartir dentro del aula de clases, las experiencias que obtuvieron en centros educativos al realizar sus prácticas profesionales.

La I-AP propicia la integración del conocimiento y la acción, junto a cada una de sus experiencias y vivencias como docente en formación, situación que es sumamente valiosa y se convierte en el objeto a ser sistematizado. Por medio de sus acciones se aplicaron la secuencia didáctica dentro del aula con relación a la experiencia de sus prácticas profesionales en los centros educativos donde tuvieron la oportunidad de desarrollar las mismas. Los 32 estudiantes de la Carrera de Educación Inicial que formaron parte de la presente investigación, fueron distribuidos en grupos de dos estudiantes por cada aula bajo la dirección de los 16 docente en ejercicio profesional de los niveles de Educación Inicial y Parvularia del Ministerio de Educación para que compartan sus experiencias y vivencias 
relacionadas el desarrollo de la metodología de I-AP. Del mismo modo, para que ellos puedan observar los procesos de enseñanza-aprendizaje que las parvularias desarrollaban con los niños de los niveles en mención y traer esa experiencia a las aulas de clase universitaria y socializarla con sus compañeros de estudio.

La UTMACH y el Centro de Investigaciones, para dar cumplimiento con los objetivos de sus líneas de investigación, considera importante la articulación de la investigación con problemas locales, regionales y nacionales, promoviendo la construcción conjunta del conocimiento entre el docente y estudiante. Lo anterior mencionado, sitúa al docente con la responsabilidad y el compromiso de buscar espacios de superación profesional en el ámbito de la investigación e innovación educativa, mediante la participación en los eventos científicos académicos organizados por la universidad, tales como: congresos, simposios, foros, donde los docentes como resultados de la investigación con sus estudiantes, presentan sus trabajos científicos.

\section{Bibliografía}

Balcázar, F. E. (2003). Investigación acción participativa (IAP): Aspectos conceptuales y dificultades de implementación. Fundamentos en humanidades, 7-8, 59-77. https://dialnet.unirioja.es/servlet/articulo?codigo $=1272956$

Balcázar, F. E., y Hernández, B. (2002). Violencia y discapacidad: Un modelo de intervención basado en la investigación-acción participativa. Psychosocial Intervention, 11(2).

Barba-Martín, R. A., Barba, J. J., y Martínez Scott, S. (2015). La formación continua colaborativa a través de la investigación-acción. Una forma de cambiar las prácticas de aula. Contextos Educativos. Revista de Educación, O(19), 161-175. https://doi.org/10.18172/con.2769

Cáceres, M., García, R., y Sánchez, L. (2002). Dificultades metodológicas y condiciones organizativas durante el desarrollo de un proceso de investigación-acción. Revista Digital de Educación y Nuevas Tecnologías Contexto Educativo, 4(22).

Carr, W., \& Kemmis, S. (2003). Becoming critical: Education knowledge and action research. Routledge.

Castañeda, J. (2013). Investigación de Acción Participativa. https://es.slideshare.net/joselyn091/accion-participativa1?next_slideshow=2

Colmenares, A. M. (2012). Investigación-acción participativa: Una metodología integradora del conocimiento y la acción. Voces y Silencios. Revista Latinoamericana de Educación, 3(1), 102-115.

Figueroa Sandoval, B., Aillon Neumann, M., Herrera Chandía, J., Yáñez Monje, V., \& Palavecino Bustos, M. (2012). "El estudio de aprendizaje», un modelo para el desarrollo del conocimiento pedagógico. Estudios pedagógicos (Valdivia), 38(2), 5568.

Kolb, D. A. (1984). Experience as the source of learning and development. Upper Sadle River: Prentice Hall.

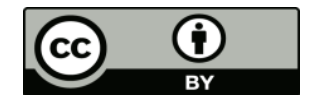

Licencia Creative Commons Atribución 4.0 Internacional (CC BY 4.0)

Revista Cátedra, 3(3), pp. 129-142, septiembre-diciembre 2020. e-ISSN:2631-2875

https://doi.org/10.29166/catedra.v3i3.2187 
Latorre, A. (2003). Investigación acción. Graó.

Lewin, K. (1946). Action research and minority problems. Journal of social issues, 2(4), 3446.

Molina Olavarría, Y. (2015). Necesidades educativas especiales, elementos para una propuesta de inclusión educativa a través de la investigación acción participativa: El caso de la Escuela México. Estudios pedagógicos (Valdivia), 41(ESPECIAL), 147-167. https://doi.org/10.4067/S0718-07052015000300010

Pereyra, M. (2008). La investigación acción en educación. Ministerio de cultura y educación de la nación, 112-118.

Pérez Gómez, Á. I., y Soto Gómez, E. (2011). Lesson study: La mejora de la práctica y la investigación docente. Cuadernos de pedagogía, 417, 64-67.

Revelo Rosero, J. E., Revuelta Domínguez, F. I., \& González-Pérez, A. (2018). Modelo de integración de la competencia digital del docente universitario para su desarrollo profesional en la enseñanza de la matemática - Universidad Tecnológica Equinoccial de Ecuador. EDMETIC, 7(1), 196-224. https://doi.org/10.21071/edmetic.v7i1.6910

Rivas Tovar, L. A. (2011). Las nueve competencias de un investigador. Investigación administrativa, $\quad$ 40(108), 34-54. http://www.scielo.org.mx/scielo.php?script=sci_abstract\&pid=S2448$76782011000200034 \& \operatorname{lng}=\mathrm{es} \& \mathrm{nrm}=\mathrm{iso} \& \ln \mathrm{ln}=\mathrm{es}$

Soto Gómez, E. S., y Pérez Gómez, Á. I. (2015). Lessons Studies: Un viaje de ida y vuelta recreando el aprendizaje comprensivo. Revista Interuniversitaria de Formación del Profesorado, 84, 15-28. 


\section{Autores}

JORGE REVELO-ROSERO obtuvo el título de Doctor (PhD) en Formación del Profesorado y TIC en Educación por la Facultad de Formación del Profesorado de la Universidad de Extremadura (España) en 2017. Obtuvo su título de Magíster en Contabilidad y Auditoría CPA en la Universidad UTE (Ecuador) en 2003. Obtuvo el título de Licenciado en Ciencias de la Educación, Profesor de Enseñanza Media en la Especialización de Matemáticas y Física por la Facultad de Filosofía, Letras y Ciencias de la Educación de la Universidad Central del Ecuador en 1993.

Actualmente es profesor titular de la Universidad UTE de Quito. Sus principales temas de investigación incluyen las áreas de administración, auditoria, contabilidad, planificación estratégica, emprendimientos, matemáticas y afines, aplicaciones matemáticas para ingeniería y física, investigación de modelos educativos con TIC. Miembro activo del Grupo de Investigación Científica GRECO-Latam. Es autor de libros y varios de capítulos de libros $\mathrm{y}$ artículos publicados en revistas de alto impacto (Emerging Source Citation Index, Latindex, Redalcy, Scielo, Scopus).

SONIA CARRILLO-PUGA obtuvo el título de Licenciada en Ciencias de la Educación, especialización Educación Inicial y Parvularia en la Universidad Técnica de Machala (Ecuador). Obtuvo el título de Magister en Gerencia Educativa en la Universidad Técnica de Machala (Ecuador).

Actualmente es profesora contratada de la Unidad Académica de Ciencias Sociales de la Universidad Técnica de Machala (Ecuador). Sus principales temas de investigación incluyen el desarrollo de estrategia metacognitivas para mejorar el nivel de comprensión lectora y hermenéutica. Es autora de varios de capítulos de libros y artículos publicados en revistas de alto impacto (Emerging Source Citation Index, Latindex).

CONSUELO REYES-CEDEÑO obtuvo el título de Licenciada en Ciencias de la Educación especialidad Filosofía y Ciencias Sociales en la Universidad Técnica de Machala (Ecuador). Magister en Docencia Universitaria e Investigación en la Universidad Técnica de Machala (Ecuador). Doctora en Currículo en la Universidad Técnica de Machala (Ecuador).

Actualmente es Docente Titular de la UTMACH, experiencia docente 30 años. Coordinadora de la Carrera de Educación Inicial. Autora de capítulos de libros, artículos científicos y ponencias.

CLARA ANDRADE-ERAZO obtuvo el título de Máster en Formación y Perfeccionamiento del Profesorado en la Universidad de Salamanca, España en 2019. Obtuvo el título de Licenciada en Ciencias de la Educación, Mención Lenguaje y Comunicación en la Universidad UTE (Ecuador) en 2012. Obtuvo el título de Licenciada en Ciencias de la Educación, Mención Ciencias Sociales en la Universidad UTE (Ecuador) en 2007.

Actualmente es docente Titular de la Unidad Educativa "Gabriela Mistral" y de la Unidad Educativa Cardenal de la Torre, de Quito (Ecuador). Autora de capítulos de libros, artículos científicos y ponencias. 